\title{
Fabrication and Physical Properties of SiC-GaAs Nano-Composites
}

\author{
G. KAlisz ${ }^{a, *}$, E. Grzanka ${ }^{a}$, D. WASIK ${ }^{b}$, \\ A. ŚWiderska-Środa ${ }^{a}$, S. Gierlotka ${ }^{a}$, J. Borysiuk $^{a}$, \\ M. KAmińsKA ${ }^{b}$, A. TWARdowski ${ }^{b}$ AND B. PAŁOSZ ${ }^{a}$ \\ ${ }^{a}$ Institute of High Pressure Physics, Polish Academy of Sciences \\ Sokołowska 29/37, 01-142 Warsaw, Poland \\ ${ }^{b}$ Institute of Experimental Physics, Warsaw University, Warsaw, Poland \\ Nano-composites consisting of primary phase of hard nanocrystalline \\ $\mathrm{SiC}$ matrix and the secondary nanocrystalline semiconductor (GaAs) phase \\ were obtained by high-pressure zone infiltration. The synthesis process oc- \\ curs in three stages: (i) at room temperature the nanopowder of $\mathrm{SiC}$ is \\ compacted along with GaAs under high pressure up to $8 \mathrm{GPa}$, (ii) the tem- \\ perature is increased above the melting point of GaAs up to $1600 \mathrm{~K}$ and, \\ the pores are being filled with liquid, (iii) upon cooling GaAs nanocrystal- \\ lites grow in the pores. Synthesis of nano-composites was performed us- \\ ing a toroid-type high-pressure apparatus (IHPP of the Polish Academy of \\ Sciences, Warsaw) and six-anvil cubic press (MAX-80 at HASYLAB, Ham- \\ burg). X-ray diffraction studies were performed using a laboratory D5000 \\ Siemens diffractometer. Phase composition, grain size, and macrostrains \\ present in the synthesized materials were examined. Microstructure of the \\ composites was characterized using scanning electron microscopy and high \\ resolution transmission electron microscopy. Far-infrared reflectivity mea- \\ surements were used to determine built-in strain.
}

PACS numbers: 81.07.-b, 07.35.+k

\section{Introduction}

Composites containing two materials with different physical properties exhibit often new properties. The composites can provide improved characteristics not obtainable by any of the original components alone and are used in a wide variety of industrial products. A common technique for manufacturing conventional composites materials, routinely used by materials scientists, are based on

${ }^{*}$ corresponding author; e-mail: kalisz@unipress.waw.pl 
infiltrating porous template made from one material (e.g., porous alumina) by another material, which is in gaseous or liquid (molten) state. The concept of various ceramic-metal and ceramic-ceramic composites has been explored in details during the last decade [1]. Infiltration of most semiconductor materials is very difficult due to their high melting temperatures and chemical reactions with the template material leading to a fast degradation of their electronic and optic properties. The attempts to create viable composite structures consisting of semiconductor particles, particularly in the nanometer grain-size, embedded in a dielectric matrix are very attractive in general, and for nano-composites in particular, due to the possibility of observing quantum confinement effects.

\section{Experimental technique}

The infiltration processes were conducted in a solid-state high-pressure cell of toroid-type $[2,3]$. The cell consists of a pair of tungsten carbide anvils and a pressure container made of limestone $\left(\mathrm{CaCO}_{3}\right)$, Fig. 1 . The sample, typically

(a)

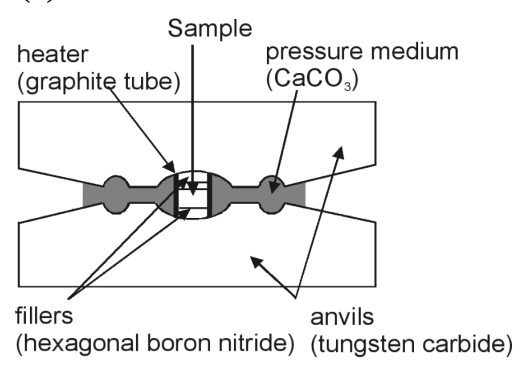

(b)

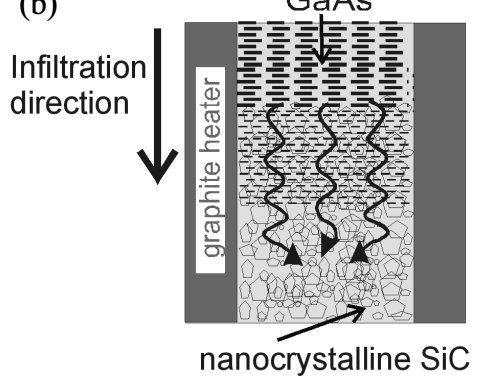

Fig. 1. Experimental configuration; (a) schematic drawing of the toroid high-pressure cell; (b) scheme of the infiltration experiment configuration.

$4 \mathrm{~mm}$ in height and $5 \mathrm{~mm}$ diameter, is placed in a graphite tube which serves as a resistance heater and is placed inside the pressure container. The remaining part is filled with hexagonal boron nitride. Uniaxial force applied to the anvils creates a quasi-isostatic pressure field in the center of the sample container. The available pressure range is 2 to $8 \mathrm{GPa}$. The lower limit is determined by the properties of the solid pressure medium, which becomes plastic only well above $1 \mathrm{GPa}$, the upper limit is determined by mechanical properties of the anvils.

The sample consists of a pre-pressed pellet of nanocrystalline SiC powder stacked with a slice of the semiconductor and placed inside the graphite heater. The pressure is gradually increased up to the pre-set value and then the heatingcooling cycle is realized. The semiconductor melts, penetrates into the pores of the compacted powder and solidifies upon cooling. Typical heating and cooling rates are of the order of $1000^{\circ} \mathrm{C} / \mathrm{min}$ and the target temperature is being kept constant for $1 \mathrm{~min}$. 
Pressure dependence of the minimum temperatures at which the infiltration occurs was determined by in situ observations using multi-anvil HP-HT apparatus MAX-80 [4] at the beamline F2.1 at the Hamburg Synchrotron Radiation Laboratory, Hamburg, Germany [5].

\section{Ceramic matrix materials}

The requirement for the matrix material is that it should form a rigid skeleton under the process pressure and temperature conditions. SiC can withstand a very large external load without excessive deformation and under pressure it sinters only above $1500^{\circ} \mathrm{C}$.

To fabricate nano-composites we used $\mathrm{SiC}$ powders of different average grain sizes: (i) 6-30 $\mathrm{nm}$ synthesized from gaseous mixtures of silane with hydrocarbons [6] and (ii) submicrometer commercial powders provided by H.C. Starck, Germany.

Packing density of as-synthesized nanopowders is typically very low, about 20-30\%. A very high pressure above $2 \mathrm{GPa}$ is required to get compacts with porosity above $50 \%$ of a solid body.

\section{Characterization of nano-composites}

Investigations of the nano-composites were performing using scanning electron microscopy (SEM) and transmission electron microscopy (TEM) observations, $\mathrm{X}$-ray diffraction analysis, and far-infrared reflectivity. Vickers microhardness measurements $\left(H V_{0.2}\right)$ were performed for selected composites.

\subsection{Microstructure and microhardness of nano-composite}

The nano-composites which we have obtained showed microstructure with two phases distributed (mixed) homogeneously on the nano-scale. Diffusion type interfaces were formed between $\mathrm{SiC}$ and GaAs, Fig. 2. The strength of the grain
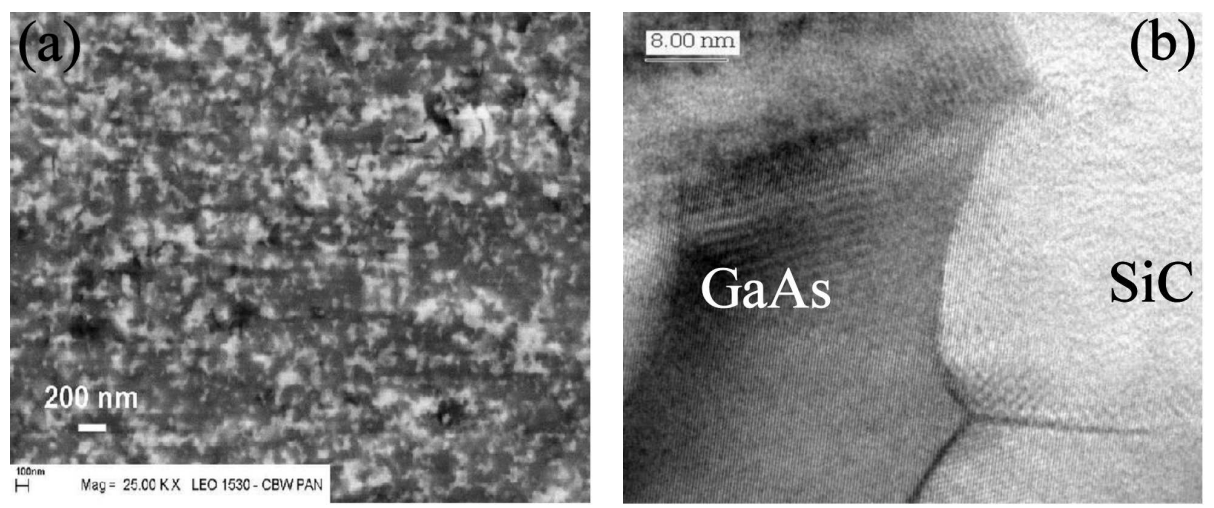

Fig. 2. Mictostructure of the $\mathrm{SiC}-\mathrm{GaAS}$ nano-composite $\left(3 \mathrm{GPa} / 1300^{\circ} \mathrm{C}\right)$; (a) SEM, (b) TEM images. 
boundaries is confirmed by very good mechanical properties of the composites. The microhardness $H V_{02}$ of $\mathrm{GaAs}-\mathrm{SiC}$ nano-composites is about $20 \mathrm{GPa}$ which is three times higher than pure $\mathrm{GaAs}-7 \mathrm{GPa}$, and $2 / 3$ of the hardness of $\mathrm{SiC}$ ceramics, about $30 \mathrm{GPa}$.

\subsection{X-ray analysis}

X-ray diffraction analysis has been used to characterize grain size and macrostrains of GaAs phase. The mean grain size of GaAs varied from 20 to $60 \mathrm{~nm}$ and depends on grain size and microstructure of the used $\mathrm{SiC}$ matrix and also on the conditions of the infiltration process. Figure 3 shows diffraction patterns of $\mathrm{SiC}-\mathrm{GaAs}$ nano-composites obtained with different $\mathrm{SiC}$ powders (sample A and $\mathrm{B}$ ) and under different pressure and temperature conditions (sample B and C). Figure 3 shows that in the composites obtained under the same conditions, with

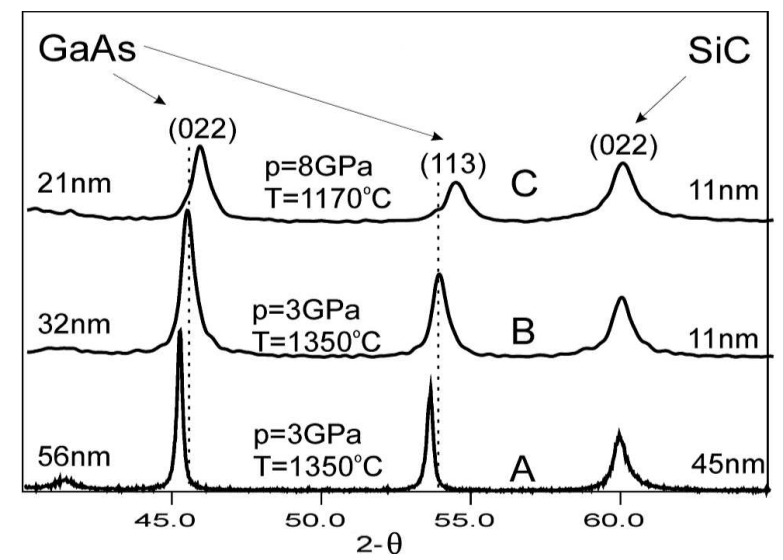

Fig. 3. X-ray diffraction patterns of $\mathrm{SiC}-\mathrm{GaAs}$ composites obtained with different $\mathrm{SiC}$ powders (sample A and B) and under different $\mathrm{p}-\mathrm{t}$ conditions (sample B and $\mathrm{C}$ ).

a decrease in the grain size of $\mathrm{SiC}$ from $45 \mathrm{~nm}$ (sample A) to $11 \mathrm{~nm}$ (sample B), the grain size of GaAs also decreases, from $56 \mathrm{~nm}$ to $32 \mathrm{~nm}$, respectively. The composites obtained under different pressure conditions have different grain sizes; the grain size of GaAs decreases from $32 \mathrm{~nm}$ (sample B) to $21 \mathrm{~nm}$ (sample C) with an increase in pressure from $3 \mathrm{GPa}$ up to $8 \mathrm{GPa}$, respectively. We can observe that diffraction lines of GaAs are shifted compared to bulk GaAs (reference dotted lines in Fig. 3), obviously due to the presence of macrostrains in the composites. The presence of macrostrains is caused by difference in compressibility and thermal expansion of $\mathrm{SiC}$ and GaAs and also depends on microstructure of the used SiC matrix. Due to complex relationships between these three factors, in relatively soft (compare to $\mathrm{SiC}$ ) GaAs there exist both tensile (sample A) and compressive (sample C) strains. 


\subsection{Infrared analysis}

Reflectivity spectra in the range of $\mathrm{SiC}$ and GaAs reststrahlen were measured by means of a Brucker IFS 113v FT-IR spectrometer. The data are shown in Fig. 4.
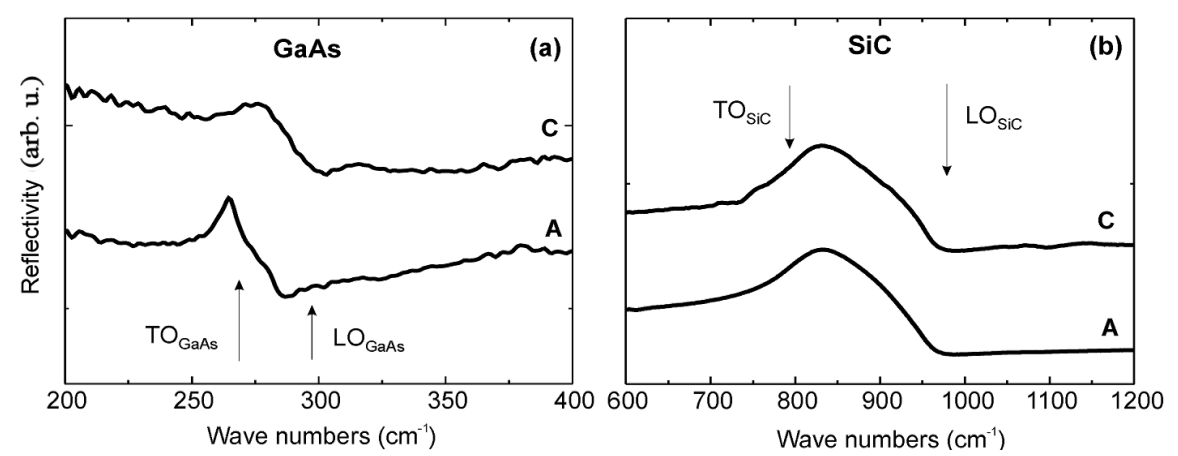

Fig. 4. Reflectivity spectra of SiC-GaAs composites (sample A and C) measured at room temperature in the range of GaAs (a) and $\mathrm{SiC}$ (b) reststrahlen domain.

They revealed that phonon energies, $\mathrm{LO}$ and $\mathrm{TO}$ of GaAs in nano-composites, are shifted (by a few $\mathrm{cm}^{-1}$ of wave numbers) with respect to bulk materials as a result of built-in strains.

\section{Summary}

We obtained a new class of materials, the semiconductor nano-composites $\mathrm{SiC}-\mathrm{GaAs}$. Through the changes of the process parameters and/or SiC matrix, HP-HT infiltration technology allow us to obtain composites with different grain sizes and different compressive or tensile macrostrains. We expect that these new materials will show electronic and optical properties different from monocrystalline GaAs and $\mathrm{SiC}$, which will be subject of the further investigations.

\section{Acknowledgments}

This work was supported by the State Committee for Scientific Research grant PBZ/KBN-03/T08A/28 (Poland). SiC powders were provided by SPAM/LFP, Laboratory "Edifices Nanometriques" from Matter Science Division, DSM, CEA, Saclay, within the Collaboration Program "Polonium".

\section{References}

[1] M. Sternitzke, J. Eur. Ceram. Soc. 17, 1061 (1997).

[2] E.A. Ekimov, A.G. Gavriliuk, B. Palosz, S. Gierlotka, P. Dłużewski, E. Tatianin, Yu. Kluev, A.M. Naletov, P. Biczyk, A. Grzegorczyk, A. Presz, Appl. Phys. Lett. 77, 954 (2000).

[3] S. Gierlotka, B. Pałosz, A. Świderska-Środa, E. Grzanka, G. Kalisz, R. Fedyk, S. Stel'makh, Solid State Phenomena 101-102, 157 (2005). 
[4] O. Shimomura, Solid State Physics under Pressure, D. Reidel Publ. Comp., Dordrecht 1985.

[5] E. Grzanka, S. Gierlotka, B. Palosz, A. Grzegorczyk, P. Biczyk, R. Pielaszek, U. Bismayer, HASYLAB Annual Report, Hamburg 2000, p. 556

[6] F. Huisken, B. Kohn, R. Alexandescu, S. Cojocaru, A. Crunteanu, C. Reynaud, G. Ledoux, J. Nanopart. Res. 1, 293 (1999). 